TRANSACTIONS OF THE

AMERICAN MATHEMATICAL SOCIETY

Volume 364, Number 9, September 2012, Pages 4589-4604

S 0002-9947(2012)05430-4

Article electronically published on April 11, 2012

\title{
ISOMORPHISM CLASSES OF SHORT GORENSTEIN LOCAL RINGS VIA MACAULAY'S INVERSE SYSTEM
}

\author{
J. ELIAS AND M. E. ROSSI
}

\begin{abstract}
Let $K$ be an algebraically closed field of characteristc zero. In this paper we study the isomorphism classes of Artinian Gorenstein local $K$ algebras with socle degree three by means of Macaulay's inverse system. We prove that their classification is equivalent to the projective classification of cubic hypersurfaces in $\mathbb{P}_{K}^{n}$. This is an unexpected result because it reduces the study of this class of local rings to the graded case. The result has applications in problems concerning the punctual Hilbert scheme $\operatorname{Hilb}_{d}\left(\mathbb{P}_{K}^{n}\right)$ and in relation to the problem of the rationality of the Poincaré series of local rings.
\end{abstract}

\section{INTRODUCTION}

The classification, up to analytic isomorphisms, of Artinian local $K$-algebras plays an important role in commutative algebra and in algebraic geometry. Among other examples, the study of the irreducibility and the smoothness of the punctual Hilbert scheme $\operatorname{Hilb}_{d}\left(\mathbb{P}_{K}^{n}\right)$ parameterizing zero-dimensional subschemes of fixed degree $d$ in $\mathbb{P}_{K}^{n}$, is strictly related to the structure of the Artinian local algebras of multiplicity $d$. In particular, because the locus of points of $\operatorname{Hilb}_{d}\left(\mathbb{P}_{K}^{n}\right)$ corresponding to Gorenstein subschemes is an open subset, often one can restrict the study to schemes which are the spectrum of Artinian Gorenstein local algebras; see for example [5, 6], 9], [10, [14, [21].

In this paper we present structure theorems of Artinian Gorenstein local $K$ algebras $(A, \mathfrak{m})$ such that $\mathfrak{m}^{4}=0$. In the main result of this paper we prove that the classification of the Artinian Gorenstein local $K$-algebras $(A, \mathfrak{m})$ with $\mathfrak{m}^{4}=0$ is equivalent to the projective classification of the cubic hypersurfaces of $\mathbb{P}_{K}^{n}$ (see Theorem 4.1 and Corollary 4.3). The key point of the paper is to prove that an Artinian Gorenstein local ring $A$ with Hilbert function $\{1, n, n, 1\}$ is isomorphic to its own associated graded ring (with respect to the maximal ideal); see Theorem 3.3 . In this case we say that $A$ is canonically graded. This is an unexpected result and it is false in general, even if the Hilbert function is symmetric. For instance there are examples of local rings with Hilbert function $\{1,2,2,2,1\}$ which are not canonically graded.

Received by the editors November 18, 2009 and, in revised form, March 22, 2010, June 29, 2010, and July 16, 2010.

2010 Mathematics Subject Classification. Primary 13H10; Secondary 13H15, 14C05.

Key words and phrases. Artinian Gorenstein local rings, inverse system, Hilbert functions, isomorphism classes.

The first author was partially supported by MTM2010-20279-C02, Acción Integrada EspañaItalia 07-09.

The second author was partially supported by M.I.U.R.: PRIN 07-09, Azione Integrata ItaliaSpagna 07-09.

(C)2012 American Mathematical Society Reverts to public domain 28 years from publication 
From classical results on the projective classification of the homogeneous cubics in $\mathbb{P}^{r}$ with $r \leq 3$, we may deduce the classification of the Artinian Gorenstein local rings with Hilbert function $\{1, m, n, 1\}$ where $n \leq 4$. This classification allows us to find geometric interpretations of the singularity of $\operatorname{Hilb}_{d}\left(\mathbb{P}^{n}\right)$ for low degrees $d$. In particular we recover recent results by Casnati and Notari; see [6, Theorem 4.1].

Local rings with low socle degree have also been studied in relation to their free resolutions. We recall that Bøgvad proved that there exist Artinian Gorenstein local rings with socle degree three and irrational Poincaré series; see [2]. However Henriques and Şega recently proved that, assuming the existence of exact zero-divisors, the Poincaré series of finitely generated modules over an Artinian Gorenstein local ring of socle degree three is rational; see [13, Theorem 4.4]. For Artinian Gorenstein local rings of socle degree three, the main result of this paper will translate the problems to Gorenstein graded $K$-algebras (see [7] for recent developments). As a consequence of this approach, taking advantage of the graded case, Corollary 3.9 recovers the quoted result by Henriques and Şega in the particular case of rings.

A central tool in this paper is Macaulay's inverse system (see [19]) which establishes a one-to-one correspondence between Artinian Gorenstein algebras and suitable polynomials. This classical correspondence has been deeply studied in the homogeneous case, among other authors, by Iarrobino in a long series of papers; see, for example, [15, [16, 17. Notice that from a categorical point of view, Macaulay's correspondence comes from Matlis duality; see [22, Theorem 5.2].

In the local case the situation is more complicated than in the graded one; nevertheless, the classification under the action of isomorphisms of algebras can be translated to the classification of the inverse system polynomials under the action of a group of transformations explicitly described. Emsalem devoted [11, Section C] to present an approach to the classification of Artinian Gorenstein local algebras by means of their inverse systems. In Section 2 we collect results spread over different papers (see [11, 15], 16, etc.) and also present explicit methods. The strategy of classifying inverse system polynomials instead of the corresponding Artinian Gorenstein local algebras has at least two advantages: first, we deal with one polynomial instead of a system of generators of the defining ideals (as in [5, 6], [9, 10]) and, second, we may perform effective computations, often reduced to a linear algebra problem (see Proposition 2.2).

We hope that this approach will be useful in studying numerical invariants of local Gorenstein singularities.

\section{INVERSE SYSTEM FOR ARTINIAN LOCAL RINGS}

Throughout this paper $K$ denotes an algebraically closed field of characteristic zero. Let $R=K \llbracket x_{1}, \ldots, x_{n} \rrbracket$ be the ring of the formal series with maximal ideal $\mathcal{M}=\left(x_{1}, \ldots, x_{n}\right)$ and let $P=K\left[y_{1}, \ldots, y_{n}\right]$ be a polynomial ring. For a positive integer $s$, we let $P_{\leq s}$ denote the set of polynomials of degree $\leq s$.

Let $I$ be an ideal of $R$ such that $R / I$ has finite length. Set $A=R / I$. We let $\mathfrak{m}=\mathcal{M}+I$ denote the maximal ideal of $A$. The socle of $A$ is the colon ideal $\left(0:_{A} \mathfrak{m}\right)$ and the socle degree $s$ of $A$ is the largest integer for which $\mathfrak{m}^{s} \neq 0$. It is well known that $A$ is Gorenstein if and only if $\operatorname{dim}_{K}(0: \mathfrak{m})=1$. From now on we assume that the embedding dimension of $A$ is $n$.

Consider $\operatorname{gr}_{\mathfrak{m}}(A):=\bigoplus_{i \geq 0} \mathfrak{m}^{i} / \mathfrak{m}^{i+1}$ the associated graded ring of $A=R / I$. It is well known that $\operatorname{gr}_{\mathfrak{m}}(A) \simeq K\left[x_{1}, \ldots, x_{n}\right] / J$ where $J$ is the homogeneous ideal 
of $K\left[x_{1}, \ldots, x_{n}\right]$ generated by the initial forms of the elements of $I$. The Hilbert function of $A$ is by definition the Hilbert function of $\operatorname{gr}_{\mathfrak{m}}(A)$, i.e.,

$$
H F_{A}(i)=\operatorname{dim}_{K}\left(\frac{\mathfrak{m}^{i}}{\mathfrak{m}^{i+1}}\right) .
$$

In this section we recall the main facts and establish notation concerning the Macaulay inverse system in the study and classification of Artinian local rings $(A, \mathfrak{m})$. The reader should refer to [11] and [16] for an extended treatment. The graded case is much better understood than the local case, and several interesting papers have been written (see for example [19, Chapter IV] and [17]).

It is known that $P$ has an $R$-module structure by means of the action

$$
\begin{aligned}
\circ: \quad R \times P & \longrightarrow P \\
(f, g) & \rightarrow f \circ g=f\left(\partial_{y_{1}}, \ldots, \partial_{y_{n}}\right)(g),
\end{aligned}
$$

where $\partial_{y_{i}}$ denotes the partial derivative with respect to $y_{i}$. If we denote by $x^{\alpha}=$ $x_{1}^{\alpha_{1}} \cdots x_{n}^{\alpha_{n}}$ and $y^{\beta}=y_{1}^{\beta_{1}} \cdots y_{n}^{\beta_{n}}$, then

$$
x^{\alpha} \circ y^{\beta}= \begin{cases}\frac{\beta !}{(\beta-\alpha) !} y^{\beta-\alpha} & \text { if } \beta_{i} \geq \alpha_{i} \text { for } i=1, \ldots, n, \\ 0 & \text { otherwise }\end{cases}
$$

where $\frac{\beta !}{(\beta-\alpha) !}=\prod_{i=1}^{n} \frac{\beta_{i} !}{\left(\beta_{i}-\alpha_{i}\right) !}$. We remark that for every $f, h \in R$ and $g \in P$, it holds $(f h) \circ g=f \circ(h \circ g)$, and we have $\mathcal{M}^{s+1} \circ g=0$ if and only if $g \in P_{\leq s}$.

Let $S \subseteq P$ be a set of polynomials. In the following we will denote by $\langle S\rangle_{K}$ the $K$-vector space generated by $S$, and by $\langle S\rangle_{R}$ the $R$-submodule of $P$ generated by $S$, i.e., the $K$-vector space generated by the elements of $S$ and by the corresponding derivatives of all orders. Starting from o, we consider the exact pairing of $K$-vector spaces:

$$
\begin{array}{ccc}
\langle,\rangle: \quad R \times P & \longrightarrow & K \\
(f, g) & \rightarrow & (f \circ g)(0) .
\end{array}
$$

For any ideal $I \subset R$, we define the following $R$-submodule of $P$ :

$$
I^{\perp}:=\{g \in P \mid\langle f, g\rangle=0 \quad \forall f \in I\}=\{g \in P \mid I \circ g=0\} .
$$

If $I=\mathcal{M}^{s+1}$, then $I^{\perp}$ coincides with $P_{\leq s}$. In general if $R / I$ has socle degree $s$, then $I^{\perp}$ is generated by polynomials of degree $\leq s$. Conversely, for every $R$-submodule $M$ of $P$ we define

$$
\operatorname{Ann}_{R}(M):=\{g \in R \mid\langle g, f\rangle=0 \quad \forall f \in M\},
$$

which is an ideal of $R$; a simple computation shows that $\operatorname{Ann}_{R}(M)=\{g \in R \mid g \circ$ $M=0\}$. If $M$ is cyclic, that is $M=\langle f\rangle=R \circ f$ with $f \in P$, then we will write $\operatorname{Ann}_{R}(f)$.

Emsalem [11, Section B, Proposition 2] and Iarrobino [16, Lemma 1.2] proved that there exists a one-to-one correspondence between ideals $I \subseteq R$ such that $R / I$ is an Artinian local ring and $R$-submodules $M$ of $P$ which are finitely generated.

Since $R / I$ and $I^{\perp}$ are finitely generated $K$-vector spaces, it is easy to see that the action $\langle$,$\rangle induces the following isomorphism of K$-vector spaces (see [11, Proposition 2 (a)]):

$$
(R / I)^{*} \simeq I^{\perp},
$$

where ()$^{*}$ denotes the dual with respect to the paring $\langle$,$\rangle induced on R / I$. 
Hence $\operatorname{dim}_{K} R / I$ (= multiplicity of $\left.R / I\right)=\operatorname{dim}_{K} I^{\perp}$. As in the graded case, it is possible to compute the Hilbert function of $A=R / I$ via the inverse system. We define the $K$-vector space

$$
\left(I^{\perp}\right)_{i}:=\frac{I^{\perp} \cap P_{\leq i}+P_{<i}}{P_{<i}} .
$$

Then, by (1), it is known that

$$
H F_{R / I}(i)=\operatorname{dim}_{K}\left(I^{\perp}\right)_{i} .
$$

Given a $K$-algebra $C$, we will denote by $\operatorname{Aut}(C)$ the group of the automorphisms of $C$ as a $K$-algebra and by $\operatorname{Aut}_{K}(C)$ a $K$-vector space. The automorphisms of $R$ as a $K$-algebra are well known. They act as replacement of $x_{i}$ by $z_{i}, i=1, \ldots, n$, such that $\mathcal{M}=\left(x_{1}, \ldots, x_{n}\right)=\left(z_{1}, \ldots, z_{n}\right)$. Actually, since $\mathcal{M}^{s+1} \subseteq I$, we are interested in the automorphisms of $R / \mathcal{M}^{s+1}$ of $K$-algebras induced by the projection $\pi$ : $R \longrightarrow R / \mathcal{M}^{s+1}$. Clearly $\operatorname{Aut}\left(R / \mathcal{M}^{s+1}\right) \subseteq \operatorname{Aut}_{K}\left(R / \mathcal{M}^{s+1}\right)$.

Let $E=\left\{e_{i}\right\}$ be the canonical basis of $R / \mathcal{M}^{s+1}$ as a $K$-vector space consisting of the standard monomials $x^{\alpha}$ ordered by the deg-lex order with $x_{1}>\cdots>x_{n}$. Then the dual basis of $E$ with respect to the perfect paring $\langle$,$\rangle is the basis E^{*}=\left\{e_{i}^{*}\right\}$ of $P_{\leq s}$ where

$$
\left(x^{\alpha}\right)^{*}=\frac{1}{\alpha !} y^{\alpha},
$$

in fact $e_{i}^{*}\left(e_{j}\right)=\left\langle e_{j}, e_{i}^{*}\right\rangle=\delta_{i j}$, where $\delta_{i j}=0$ if $i \neq j$ and $\delta_{i i}=1$. Hence, for any $\varphi \in \operatorname{Aut}_{K}\left(R / \mathcal{M}^{s+1}\right)$, we may associate a matrix $M(\varphi)$ with respect to the basis $E$ of size $r=\operatorname{dim}_{K}\left(R / \mathcal{M}^{s+1}\right)=\left(\begin{array}{c}n+s \\ s\end{array}\right)$. We have the following natural sequence of morphisms of groups:

$$
\operatorname{Aut}(R) \stackrel{\pi}{\longrightarrow} \operatorname{Aut}\left(R / \mathcal{M}^{s+1}\right) \stackrel{\sigma}{\longrightarrow} \operatorname{Aut}_{K}\left(R / \mathcal{M}^{s+1}\right) \stackrel{\rho_{E}}{\longrightarrow} \mathrm{Gl}_{r}(K) .
$$

Given $I$ and $J$ ideals of $R$ such that $\mathcal{M}^{s+1} \subset I, J$, there exists an isomorphism of $K$-algebras

$$
\varphi: R / I \rightarrow R / J
$$

if and only if $\varphi$ is canonically induced by a $K$-algebra automorphism of $R / \mathcal{M}^{s+1}$ sending $I / \mathcal{M}^{s+1}$ to $J / \mathcal{M}^{s+1}$. In particular $\varphi$ is an isomorphism of $K$-vector spaces. Dualizing

$$
\varphi^{*}:(R / J)^{*} \rightarrow(R / I)^{*}
$$

is an isomorphism of the $K$-vector subspaces $(R / I)^{*} \simeq I^{\perp}$ and $(R / J)^{*} \simeq J^{\perp}$ of $P_{\leq s}$. Hence ${ }^{t} M(\varphi)$ is the matrix associated to $\varphi^{*}$ with respect to the basis $E^{*}$ of $P_{\leq s}$.

We can complete (4) by the following commutative diagram which helps to visualize our setting:

$$
\begin{array}{ccc}
\operatorname{Aut}_{K}\left(R / \mathcal{M}^{s+1}\right) & \stackrel{\rho_{E}}{\longrightarrow} & \mathrm{Gl}_{r}(K) \\
\downarrow * & & \downarrow{ }^{t}() \\
\operatorname{Aut}_{K}\left(P_{\leq s}\right) & \stackrel{\rho_{E^{*}}}{\longrightarrow} & \mathrm{Gl}_{r}(K) .
\end{array}
$$

We denote by $\mathcal{R}$ the subgroup of $\operatorname{Aut}_{K}\left(P_{\leq s}\right)$ (automorphisms of $P_{\leq s}$ as a $K$ vector space) represented by the matrices ${ }^{t} M(\varphi)$ of $\mathrm{Gl}_{r}(K)$ with $\varphi \in \operatorname{Aut}\left(R / \mathcal{M}^{s+1}\right)$.

Theorem 2.1 ([11, Proposition 15]). The classification, up to analytic isomorphism, of the Artinian local $K$-algebras of multiplicity $d$, socle degree $s$ and embedding dimension $n$ is equivalent to the classification, up to the action of $\mathcal{R}$, of 
the $K$-vector subspaces of $P_{\leq s}$ of dimension $d$, stable by derivations and containing $P_{\leq 1}=K\left[y_{1}, \ldots, y_{n}\right]_{\leq 1}$.

More precise results can be stated for Artinian Gorenstein local $K$-algebras. A local ring $A=R / I$ is an Artinian Gorenstein local ring of socle degree $s$ if and only if its dual module $I^{\perp}$ is a cyclic $R$-submodule of $P$ generated by a polynomial $F \in P$ of degree $s$ (see also [18, Theorem 220] and [16, Lemma 1.2]). We will denote by $A_{F}$ the Gorenstein Artin algebra associated to $F \in P$, i.e.,

$$
A_{F}=R / \operatorname{Ann}_{R}(F) \text {. }
$$

Hence each Artinian Gorenstein local ring of socle $s$ will be equipped with a polynomial $F \in P$ of degree $s$. The polynomial $F$ is not unique, but is determined up to a unit $u$ of $R$ ( $F$ can be replaced by $u \circ F)$.

Our goal is to translate the classification of the Artinian Gorenstein local rings $A=R / I$ of socle $s$ in terms of the corresponding polynomials of degree $s$ in $P$.

Let $\varphi \in \operatorname{Aut}\left(R / \mathcal{M}^{s+1}\right)$. From the previous facts we have

$$
\varphi\left(A_{F}\right)=A_{G} \text { if and only if }\left(\varphi^{*}\right)^{-1}\left(\langle F\rangle_{R}\right)=\langle G\rangle_{R} .
$$

It is easy to verify that

(6) $\varphi\left(A_{F}\right)=A_{G}$ if and only if $\left(\varphi^{*}\right)^{-1}(F)=u \circ G$ where $u$ is a unit in $R$.

Let $u$ be an invertible element of $R / \mathcal{M}^{s+1}$. The corresponding action of $u$ in $P_{<s}$ is a $K$-vector space isomorphism, so we can consider the associated matrix $N(u) \in \mathrm{Gl}_{r}(K)$ with respect to the basis $E^{*}$. If $F=b_{1} e_{1}^{*}+\cdots+b_{r} e_{r}^{*} \in P_{\leq s}$, then we will denote the row vector of the coefficients of the polynomial with respect to the basis $E^{*}$ by

$$
[F]_{E^{*}}=\left(b_{1}, \ldots, b_{r}\right)
$$

Hence from (6) we deduce the following key result:

Proposition 2.2. The Artinian Gorenstein local rings $A_{F}$ and $A_{G}$ of socle degree $s$ are isomorphic if and only if there are $\varphi \in \operatorname{Aut}\left(R / \mathcal{M}^{s+1}\right)$ and an invertible element $u \in R / \mathcal{M}^{s+1}$ such that

$$
[G]_{E^{*}}\left({ }^{t} N(u) M(\varphi)\right)=[F]_{E^{*}} .
$$

The above result enables us to study the isomorphism classes of Artinian Gorenstein algebras in an effective computational framework. The strategy of this paper is to classify the Artinian local algebras by classifying their inverse systems by means of (77). Notice that the Proposition [2.2 result extends [17, Appendix A] or [1, Section C, Proposition 17] to the nonhomogeneous case.

We say that $F \in P=K\left[y_{1}, \ldots, y_{n}\right]$ is nondegenerate if the embedding dimension of the Gorenstein algebra $A_{F}$ is $n$, i.e., $H F_{A_{F}}(1)=n$. Hence a polynomial $F$ of degree $s$ is nondegenerate if and only if the dimension of the $K$-vector space of the derivatives of order $s-1$ of $F$ is $n$ or, equivalently, the ideal $\operatorname{Ann}_{R}(F)$ does not contain elements of valuation one.

In order to classify the Artinian Gorenstein local rings of given multiplicity, we need information on the admissible Hilbert functions. In the graded case, the Hilbert function of an Artinian Gorenstein algebra is symmetric. Little is known about the Hilbert function in the local case. The problem comes from the fact that, in general, the associated algebra $G=\operatorname{gr}_{\mathfrak{m}}(A)=\bigoplus_{n \geq 0} \mathfrak{m}^{n} / \mathfrak{m}^{n+1}$ is no longer Gorenstein. 
Nevertheless, Iarrobino in [16 proved interesting results concerning $G$. Let us consider a filtration of $G$ by a descending sequence of ideals

$$
G=C(0) \supseteq C(1) \supseteq \cdots \supseteq C(s-2)
$$

whose successive quotient

$$
Q(a)=C(a) / C(a+1)
$$

are reflexive graded $G$-modules of socle degree $\frac{s-a}{2}(s=$ socle-degree of $A)$; see [16, Theorem 1.5]. Hence $Q(a)$ has symmetric Hilbert function.

In particular $Q(0)=G / C(1)$ is the unique (up to isomorphism) graded Gorenstein quotient of $G$ with the same socle degree $s$. Iarrobino proved that if $H F_{A}(n)$ is symmetric, then $G=Q(0)$ and it is Gorenstein. The same result has also been proved in a different manner by J. Watanabe in [23]. Hence if $A$ is Gorenstein ([16, Proposition 1.7] and [11, Proposition 7])

$$
G \text { is Gorenstein } \Longleftrightarrow H F_{A}(n) \text { is symmetric } \Longleftrightarrow G=Q(0) \text {. }
$$

The $G$-module $Q(0)$ plays a crucial role, and it can be computed in terms of the corresponding polynomial in the inverse system. Let $F \in P$ be a polynomial of degree $s$ and denote by $F_{s}$ the form of highest degree in $F$, that is, $F=F_{s}+\cdots$ terms of lower degree, then

$$
Q(0) \simeq R / \operatorname{Ann}_{R}\left(F_{s}\right)
$$

see [11, Proposition 7] and [16, Lemma 1.10].

\section{Artinian Gorenstein algebras with $H F_{A}=\{1, n, n, 1\}$}

Let $I$ be an ideal of $R=k\left[\left[x_{1}, \ldots, x_{n}\right]\right]$. Assume $A=R / I$ is an Artinian Gorenstein local ring with Hilbert function $H F_{A}=\{1, n, n, 1\}$. This means that the socle degree is three and $H F_{A}(1)=H F_{A}(2)=n, H F_{A}(0)=H F_{A}(3)=1$. As we have seen, since $H F_{A}$ is symmetric, then $G=\operatorname{gr}_{\mathfrak{m}}(A)$ is Gorenstein. We recall that $G=K\left[x_{1}, \ldots, x_{n}\right] / J$, where $J$ is the ideal generated by the initial forms of the elements of $I$. Since $A$ is Artinian, there is a natural isomorphism between $G$ and $R / J R$. In this section we prove that there exists an isomorphism of local rings between $A$ and $G$ (actually between $A$ and $R / J R$ ); see Theorem 3.3 .

It is very rare that a local ring is isomorphic to its associated graded ring. Following Emsalem [11, we say that $A$ is canonically graded if $A \simeq G$. Gorenstein local rings with symmetric Hilbert function are not necessarily canonically graded. The following example shows that we cannot extend the main result of this section to higher socle degrees without new assumptions.

Example 3.1. Let $A$ be an Artinian Gorenstein local $K$-algebra with Hilbert function $H F_{A}=\{1,2,2,2,1\}$. Then $A$ is isomorphic to one and only one of the following rings:

(a) $R / I$ with $I=\left(x_{1}^{4}, x_{2}^{2}\right) \subseteq R=K\left[\left[x_{1}, x_{2}\right]\right]$, and $I^{\perp}=\left\langle y_{1}^{3} y_{2}\right\rangle$. In this case $A$ is canonically graded.

(b) $R / I$ with $I=\left(x_{1}^{4},-x_{1}^{3}+x_{2}^{2}\right) \subseteq R=K\left[\left[x_{1}, x_{2}\right]\right]$, and $I^{\perp}=\left\langle y_{1}^{3} y_{2}+y_{2}^{3}\right\rangle$. The associated graded ring is of type $(a)$ and it is not isomorphic to $R / I$. Hence $A$ is not canonically graded.

(c) $R / I$ with $I=\left(x_{1}^{2}+x_{2}^{2}, x_{2}^{4}\right) \subseteq R=K\left[\left[x_{1}, x_{2}\right]\right]$, and $I^{\perp}=\left\langle y_{1} y_{2}\left(y_{1}^{2}-y_{2}^{2}\right)\right\rangle$. In this case $A$ is canonically graded. 
The computation can be performed by using Proposition 2.2, a different approach can be found in $[9]$.

Since $G$ is a graded Gorenstein algebra of embedding dimension $n$, it will be useful to get information on the homogeneous cubics $F \in P=K\left[y_{1}, \ldots, y_{n}\right]$ such that $H F_{A_{F}}=\{1, n, n, 1\}$, that is, to characterize the homogeneous cubics which are nondegenerate.

Remark 3.2. We consider a homogeneous form $F_{3} \in P=K\left[y_{1}, \ldots, y_{n}\right]$ of degree three. We write $F_{3}$ in the dual basis $E^{*}$

$$
F_{3}=\sum_{|\underline{\mid}|=3} \alpha_{\underline{i}} \frac{1}{i \underline{i} !} y^{\underline{i}} .
$$

$F_{3}$ is nondegenerate if and only if the $K$-vector space generated by all the derivatives of order two has dimension $n$, that is,

$$
\left\langle\partial_{\underline{i}} F_{3}:|\underline{i}|=2\right\rangle_{K}=P_{1} .
$$

This condition can be formulated in terms of the rank of a matrix, say $\Delta_{F_{3}}$, given by the coefficients of the linear forms $\partial_{\underline{i}} F_{3},|\underline{i}|=2$. The matrix $\Delta_{F_{3}}$ has size $n \times\left(\begin{array}{c}n+1 \\ 2\end{array}\right)$ with entries in the $\alpha_{\underline{i}}^{\prime} s \in K$. We label the rows by $j=1, \ldots, n$, and the columns by $\underline{i} \in \mathbb{N}^{n},|\underline{i}|=2$. We have

$$
\left(\Delta_{F_{3}}\right)_{j, \underline{i}}=\alpha_{\underline{i}+\delta_{j}}
$$

where $\delta_{j}$ is the $n$-tuple with zero entries but 1 in position $j$, hence $\underline{i}+\delta_{j}=$ $\left(i_{1}, \ldots, i_{j}+1, \ldots, i_{n}\right)$. In fact we have

$$
\partial_{\underline{i}} F_{3}=\sum_{|\underline{p}|=3} \alpha_{\underline{p}} y^{\underline{p}-\underline{i}}=\sum_{j=1}^{n} \alpha_{\underline{i}+\delta_{j}} y_{j} .
$$

Hence $F_{3}$ is nondegenerate if and only if $\operatorname{rk}\left(\Delta_{F_{3}}\right)=n$.

Theorem 3.3. Let $A$ be an Artinian Gorenstein local $K$-algebra with Hilbert function $\{1, n, n, 1\}$. Then $A$ is canonically graded.

Proof. Let $A=R / I$ with $R=K\left[\left[x_{1}, \ldots, x_{n}\right]\right]$ and let $F=F_{0}+F_{1}+F_{2}+F_{3}$ be a polynomial of $P=K\left[y_{1}, \ldots, y_{n}\right]$ of degree three such that $I=\operatorname{Ann}_{R}(F)$ ( $F_{i}$ denotes the homogeneous components of degree $i$ ). Since $H F_{A}$ is symmetric, then $G=\operatorname{gr}_{\mathfrak{m}}(A)$ is Gorenstein, in particular $G=Q(0) \simeq R / \operatorname{Ann}_{R}\left(F_{3}\right)=A_{F_{3}}$ and $\operatorname{rk}\left(\Delta_{F_{3}}\right)=n$ being $F_{3}$ nondegenerate for the Hilbert function $\{1, n, n, 1\}$. By the admissibility of $F_{3}$ we deduce that $P_{\leq 1} \subseteq\left\langle F_{2}+F_{3}\right\rangle_{R}$. Hence we may assume $F=F_{3}+F_{2}$, that is,

$$
I^{\perp}=\langle F\rangle_{R}=\left\langle F_{2}+F_{3}\right\rangle_{R} .
$$

So we have to prove that, however we fix $F_{2}$, there exists an automorphism $\varphi$ of $R / \mathcal{M}^{4}$ which induces

$$
A_{F_{3}} \simeq A_{F_{2}+F_{3}} .
$$

Let $\varphi$ be an automorphism of $R / \mathcal{M}^{4}$ with the identity as Jacobian defined as

$$
\varphi\left(x_{j}\right)=x_{j}+\sum_{|\underline{i}|=2} a_{\underline{i}}^{j} x^{\underline{i}}
$$


for $j=1, \ldots, n$. We prove that there exists $\underline{a}=\left(a_{\underline{i}}^{1},|\underline{i}|=2 ; \ldots ; a_{\underline{i}}^{n},|\underline{i}|=2\right) \in$ $K^{n\left(\begin{array}{c}n+1 \\ 2\end{array}\right)}$, the row vector of the coefficients defining $\varphi$, such that

$$
\left[F_{3}\right]_{E^{*}} M(\varphi)=\left[F_{2}+F_{3}\right]_{E^{*}}
$$

The matrix associated to $\varphi$, say $M(\varphi)$, is an element of $\mathrm{Gl}_{r}(K), r=\left(\begin{array}{c}n+3 \\ 4\end{array}\right)$, with respect to the basis $E$ of $R / \mathcal{M}^{4}$, hence

$$
M(\varphi)=\left(\begin{array}{l|l|l|l}
1 & 0 & 0 & 0 \\
\hline 0 & I_{n} & 0 & 0 \\
\hline 0 & D & I_{\left(\begin{array}{c}
n+1 \\
2
\end{array}\right)} & 0 \\
\hline 0 & 0 & \mathrm{~B} & I_{\left(\begin{array}{c}
n+2 \\
3
\end{array}\right)}
\end{array}\right),
$$

where for all $t \geq 1, I_{t}$ denotes the $t \times t$ identity matrix. The first block column corresponds to the image $\varphi(1)=1$; the second block column corresponds to the image of $\varphi\left(x_{i}\right), i=1, \ldots, n$; the third block column corresponds to the image of $\varphi\left(x^{\underline{i}}\right)$ such that $|\underline{i}|=2$; and finally the last block column corresponds to the image of $\varphi\left(x^{\underline{i}}\right)$ such that $|\underline{i}|=3$, i.e., the identity matrix.

Hence $D$ is the $\left(\begin{array}{c}n+1 \\ 2\end{array}\right) \times n$ matrix defined by the coefficients of the degree two monomials of $\varphi\left(x_{i}\right), i=1, \ldots, n$ and $B$ is the $\left(\begin{array}{c}n+2 \\ 3\end{array}\right) \times\left(\begin{array}{c}n+1 \\ 2\end{array}\right)$ matrix defined by the coefficients of the degree three monomials appearing in $\varphi\left(x^{\underline{i}}\right),|\underline{i}|=2$. It is clear that $M(\varphi)$ is determined by $D$, and the entries of $B$ are linear forms in the variables $a_{\underline{i}}^{j}$, with $|\underline{i}|=2, j=1, \ldots, n$. Let

$$
F_{2}=\sum_{|\underline{i}|=2} \beta_{\underline{i}} \frac{1}{\underline{i} !} y^{\underline{i}} \text { and } F_{3}=\sum_{|\underline{i}|=3} \alpha_{\underline{i}} \frac{1}{\underline{i} !} y^{\underline{i}} .
$$

Hence (9) is equivalent to the equality

$$
\left[\alpha_{\underline{i}}\right] B=\left[\beta_{\underline{i}}\right] .
$$

Then we get a system of $\left(\begin{array}{c}n+1 \\ 2\end{array}\right)$ equations which are bihomogeneous polynomials in the $\left\{\alpha_{\underline{i}}\right\}$ and $\underline{a} \in K^{n\left(\begin{array}{c}n+1 \\ 2\end{array}\right)}$ of bidegree $(1,1)$. Then there exists a matrix $M_{F_{3}}$ of size $\left(\begin{array}{c}n+1 \\ 2\end{array}\right) \times n\left(\begin{array}{c}n+1 \\ 2\end{array}\right)$ and entries in the $\left\{\alpha_{\underline{i}}\right\}^{\prime} s$ such that

$$
{ }^{t}\left(\left[\alpha_{\underline{i}}\right] B\right)=M_{F_{3}}{ }^{t} \underline{a},
$$

where ${ }^{t} \underline{a}$ denotes the transpose of the row vector $\underline{a}$. We have to prove that the following linear system in $\left(\begin{array}{c}n+1 \\ 2\end{array}\right)$ equations and the $n\left(\begin{array}{c}n+1 \\ 2\end{array}\right)$ indeterminates $\underline{a}=$ $\left(a_{\underline{i}}^{1} ; \ldots ; a_{\underline{i}}^{n}\right)$

$$
M_{F_{3}}{ }^{t} \underline{a}={ }^{t}\left[\beta_{\underline{i}}\right]
$$

is compatible. The result follows if we show that $\operatorname{rk}\left(M_{F_{3}}\right)$ is maximal, i.e., $\operatorname{rk}\left(M_{F_{3}}\right)$ $=\left(\begin{array}{c}n+1 \\ 2\end{array}\right)$. 
Claim. The matrix $M_{F_{3}}$ has the upper-diagonal structure

$M_{F_{3}}=\left(\begin{array}{l|l|l|l|l}M_{F_{3}}^{1} & * & \ldots & * & * \\ \hline 0 & M_{F_{3}}^{2} & \ldots & * & * \\ \hline \vdots & \vdots & \vdots & \vdots & \vdots \\ \hline 0 & 0 & 0 & M_{F_{3}}^{n-1} & * \\ \hline 0 & 0 & 0 & 0 & M_{F_{3}}^{n}\end{array}\right)$,

where $M_{F_{3}}^{l}$ is an $(n-l+1) \times\left(\begin{array}{c}n+1 \\ 2\end{array}\right)$ matrix, $l=1, \ldots, n$, such that

(i) 1-st row of $M_{F_{3}}^{1}=2$ times the 1-st row of $\Delta_{F_{3}}$,

$t$-th row of $M_{F_{3}}^{1}=t$-th row of $\Delta_{F_{3}}, t=2, \ldots, n$.

(ii) 1-st row of $M_{F_{3}}^{l}=2$ times the $l$-st row of $\Delta_{F_{3}}$, for $l=2, \ldots, n$,

$t$-th row of $M_{F_{3}}^{l}=(l+t-1)$-th row of $\Delta_{F_{3}}$, for $t=2, \ldots, n-l+1$, $l=2, \ldots, n$,

where $\Delta_{F_{3}}$ is the matrix defined in Remark 3.2 of the coefficients of the second derivatives of $F_{3}$.

Proof of the Claim. Let us recall that the entries of the columns of $B$ are the coefficients of the degree three monomials of the support of $\varphi\left(x^{\underline{i}}\right),|\underline{i}|=2$. Hence the entries of the $a_{i}^{l}$-th column of $M_{F_{3}}$ are the coefficients of the terms of degree three in the support of $F_{3}$ which appear in $\varphi\left(x^{\underline{i}}\right)$ with coefficient $a_{\underline{i}}^{l}$. Given integers $1 \leq l \leq j \leq n$, let us compute $\varphi\left(x_{l} x_{j}\right)$. If $l \neq j$, then

$$
\varphi\left(x_{l} x_{j}\right)=x_{l} x_{j}+\sum_{|\underline{i}|=2} a_{\underline{i}}^{j} x^{\underline{i}} x_{l}+\sum_{|\underline{i}|=2} a_{\underline{i}}^{l} x^{\underline{i}} x_{j}+\text { terms of degree } 4 .
$$

Since $x^{\underline{i}} x_{j}=x^{\underline{i}+\delta_{j}}$ and $j>l$, we get

$$
\left(M_{F_{3}}\right)_{\delta_{l}+\delta_{j}, a_{\underline{i}}^{l}}=\alpha_{\underline{i}+\delta_{j}}
$$

and

$$
\left(M_{F_{3}}\right)_{\delta_{l}+\delta_{j}, a_{\underline{i}}^{j}}=\alpha_{\underline{i}+\delta_{l}} .
$$

If $j=l$, then

$$
\varphi\left(x_{l}^{2}\right)=x_{l}^{2}+2 \sum_{|\underline{i}|=2} a_{\underline{\underline{i}}}^{l} x^{\underline{i}} x_{l}+\text { terms of degree } 4
$$

so

$$
\left(M_{F_{3}}\right)_{2 \delta_{l}, a_{\underline{i}}^{l}}=2 \alpha_{\underline{i}+\delta_{l}} .
$$

Hence the row $\left(\delta_{l}+\delta_{j}\right)$-th of $M_{F_{3}}, j>l$, can be split in two nonzero subsets of entries. The first subset, with respect to the lex ordering, corresponds to the columns $a_{\underline{i}}^{l},|\underline{i}|=2$, with entries $\alpha_{\underline{i}+\delta_{j}}$, the second subset of entries corresponds to the column $a_{i}^{j}$ with entries $\alpha_{\underline{i}+\delta_{l}}$. From these facts we get the upper-diagonal block structure of $M_{F_{3}}$. In particular, if we fix $l=1, \ldots, n$, the matrices $M_{F_{3}}^{l}, l=1, \ldots, n$ appearing in the claim are determined by the columns $a_{\underline{i}}^{l}$ and the rows $\delta_{l}+\delta_{j}$ with $l \leq j \leq n((n-j+1)$-rows $)$ and

$$
\left(M_{F_{3}}^{l}\right)_{\delta_{l}+\delta_{j}, a_{\underline{i}}^{l}}=\alpha_{\underline{i}+\delta_{j}} \quad \text { if } l>j, \quad\left(M_{F_{3}}^{l}\right)_{\delta_{l}+\delta_{j}, a_{\underline{i}}^{l}}=2 \alpha_{\underline{i}+\delta_{j}} \quad \text { if } l=j .
$$


By Remark 3.2 and equation (8) we get

$$
\left(M_{F_{3}}^{l}\right)_{\delta_{l}+\delta_{j}, a_{\underline{i}}^{l}}=\left(\Delta_{F_{3}}\right)_{j, \underline{i}} \quad \text { if } l>j, \quad\left(M_{F_{3}}^{l}\right)_{\delta_{l}+\delta_{j}, a_{\underline{i}}^{l}}=2\left(\Delta_{F_{3}}\right)_{j, \underline{i}} \quad \text { if } l=j
$$

as claimed.

Now we prove that

$$
\operatorname{rk}\left(M_{F_{3}}\right)=\left(\begin{array}{c}
n+1 \\
2
\end{array}\right) \text {. }
$$

Since $F_{3}$ is nondegenerate, by Remark 3.2 we have $\operatorname{rank}\left(\Delta_{F_{3}}\right)=n$. Now $M_{F_{3}}^{l}$ for $l=1, \ldots, n$ is a matrix of size $n-l+1 \times\left(\begin{array}{c}n+1 \\ 2\end{array}\right)$ obtained by $\Delta_{F_{3}}$ by deleting the first $l$ rows. Hence $\operatorname{rk}\left(M_{F_{3}}^{l}\right)=n-l+1$ and the result follows.

From the previous result, we easily get the following consequences.

Corollary 3.4. There exists an isomorphism between the Artinian Gorenstein local $K$-algebras $(A, \mathfrak{m})$ and $(B, \mathfrak{n})$ with Hilbert function $\{1, n, n, 1\}$ if and only if $\operatorname{gr}_{\mathfrak{m}}(A) \simeq \operatorname{gr}_{\mathfrak{n}}(B)$ as graded $K$-algebras.

Corollary 3.5. The classification of Artinian Gorenstein local K-algebras with Hilbert function $H F_{A}=\{1, n, n, 1\}$ is equivalent to the projective classification of the hypersurfaces $V(F) \subset \mathbb{P}_{K}^{n-1}$, where $F$ is a degree three nondegenerate form in $n$ variables.

The classification of the Artinian Gorenstein local rings with Hilbert function $H F_{A}=\{1, n, n, 1\}$ for $1 \leq n \leq 3$ has been studied by Casnati and Notari in [6, Theorem 4.1] and by Cartwright et al. in [4. By using Corollary 3.5, the problem can be reduced to the homogeneous case which is well known for $1 \leq n \leq 3$. Hence we can describe the geometric models of the varieties defined by them.

If $n=1$, then it is clear that $A \cong K[[x]] /\left(x^{4}\right)$, so there is only one analytic model.

Proposition 3.6. Let $A$ be an Artinian Gorenstein local $K$-algebra with Hilbert function $H F_{A}=\{1,2,2,1\}$. Then $A$ is isomorphic to one and only one of the following quotients of $R=K\left[\left[x_{1}, x_{2}\right]\right]$.

\begin{tabular}{|c|c|c|}
\hline Model $A=R / I$ & Inverse system $F$ & Geometry of $C=V(F) \subset \mathbb{P}_{K}^{1}$ \\
\hline$\left(x_{1}^{3}, x_{2}^{2}\right)$ & $y_{1}^{2} y_{2}$ & Double point plus a simple point \\
\hline$\left(x_{1} x_{2}, x_{1}^{3}-x_{2}^{3}\right)$ & $y_{1}^{3}-y_{2}^{3}$ & Three distinct points \\
\hline
\end{tabular}

Proof. Let us assume $n=2$, then $\operatorname{gr}_{\mathfrak{m}}(A)=K\left[y_{1}, y_{2}\right] / \operatorname{Ann}(F)$ where $F \in K\left[y_{1}, y_{2}\right]$ is a degree three form on two variables $y_{1}, y_{2}$. Since $K$ is an algebraic closed field, $F$ can be decomposed as product of three linear forms $L_{1}, L_{2}, L_{3}$, i.e., $F=L_{1} L_{2} L_{3}$. We set $d=\operatorname{dim}_{K}\left\langle L_{1}, L_{2}, L_{3}\right\rangle$, so we only have to consider three cases. If $d=1$, then we can assume $F=y_{1}^{3}$, but this case does not occur because $F$ is degenerated. If $d=2$, then we can assume $F=y_{1}^{2} y_{2}$. It is easy to see that $\operatorname{Ann}\left(\left\langle y_{1}^{2} y_{2}\right\rangle\right)=\left(x_{1}^{3}, x_{2}^{2}\right)$. If $d=3$, then we can assume $F=y_{1}^{3}-y_{2}^{3}$. In this case we get $\operatorname{Ann}\left(\left\langle y_{1}^{3}-y_{2}^{3}\right\rangle\right)=$ $\left(x_{1} x_{2}, x_{1}^{3}-x_{2}^{3}\right)$. Since $V\left(y_{1}^{2} y_{2}\right)$ (resp. $\left.V\left(y_{1}^{3}-y_{2}^{3}\right)\right)$ is a degree three subscheme of $\mathbb{P}_{K}^{1}$ with two (resp. three) point basis, we deduce that the algebras of the statement are not isomorphic. 
We know that any plane elliptic cubic curve $C \subset \mathbb{P}_{K}^{2}$ is defined, in a suitable system of coordinates, by a Legendre's equation

$$
L_{\lambda}=y_{2}^{2} y_{3}-y_{1}\left(y_{1}-y_{3}\right)\left(y_{1}-\lambda y_{3}\right)
$$

with $\lambda \neq 0,1$. This equation is equipped with the $j$-invariant

$$
j(\lambda)=2^{8} \frac{\left(\lambda^{2}-\lambda+1\right)}{\lambda^{2}(\lambda-1)^{2}} .
$$

It is well known that two plane elliptic cubic curves $C_{i}=V\left(L_{\lambda_{i}}\right) \subset \mathbb{P}_{K}^{2}, i=1,2$, are projectively isomorphic if and only if $j\left(\lambda_{1}\right)=j\left(\lambda_{2}\right)$; see [12].

Proposition 3.7. Let $A$ be an Artinian Gorenstein local $K$-algebra with Hilbert function $H F_{A}=\{1,3,3,1\}$. Then $A$ is isomorphic to one and only one of the following quotients of $R=K\left[\left[x_{1}, x_{2}, x_{3}\right]\right]$ :

\begin{tabular}{|c|c|c|}
\hline Model $A=R / I$ & Inverse system $F$ & Geometry of $C=V(F) \subset \mathbb{P}_{K}^{2}$ \\
\hline$\left(x_{1}^{2}, x_{2}^{2}, x_{3}^{2}\right)$ & $y_{1} y_{2} y_{3}$ & Three independent lines \\
\hline$\left(x_{1}^{2}, x_{1} x_{3}, x_{3} x_{2}^{2}, x_{2}^{3}, x_{3}^{2}+x_{1} x_{2}\right)$ & $y_{2}\left(y_{1} y_{2}-y_{3}^{2}\right)$ & Conic and a tangent line \\
\hline$\left(x_{1}^{2}, x_{2}^{2}, x_{3}^{2}+6 x_{1} x_{2}\right)$ & $x_{3}\left(x_{1} x_{2}-x_{3}^{2}\right)$ & Conic and a nontangent line \\
\hline$\left(x_{3}^{2}, x_{1} x_{2}, x_{1}^{2}+x_{2}^{2}-3 x_{1} x_{3}\right)$ & $y_{2}^{2} y_{3}-y_{1}^{2}\left(y_{1}+y_{3}\right)$ & Irreducible nodal cubic \\
\hline$\left(x_{3}^{2}, x_{1} x_{2}, x_{1} x_{3}, x_{2}^{3}, x_{1}^{3}+3 x_{2}^{2} x_{3}\right)$ & $y_{2}^{2} y_{3}-y_{1}^{3}$ & Irreducible cuspidal cubic \\
\hline$\left(x_{2} x_{3}, x_{1} x_{3}, x_{1} x_{2}, x_{2}^{3}-x_{3}^{3}, x_{1}^{3}-x_{3}^{3}\right)$ & $y_{1}^{3}+y_{2}^{3}+y_{3}^{3}$ & Elliptic Fermat curve \\
\hline$I(\lambda)=\left(x_{1} x_{2}, H_{1}, H_{2}\right)$ & $L_{\lambda}, j(\lambda) \neq 0$ & Elliptic non-Fermat curve \\
\hline
\end{tabular}

with $H_{1}=\lambda^{2} x_{1}^{2}+\lambda(1+\lambda) x_{1} x_{3}+\left(\lambda^{2}-\lambda+1\right) x_{3}^{2}, H_{2}=\lambda^{2} x_{2}^{2}+\lambda x_{1} x_{3}+(1+\lambda) x_{3}^{2}$, and $I\left(\lambda_{1}\right) \cong I\left(\lambda_{2}\right)$ if and only if $j\left(\lambda_{1}\right)=j\left(\lambda_{2}\right)$.

Proof. Let us assume that $F$ is the product of the linear forms $l_{1}, l_{2}, l_{3}$. If $l_{1}, l_{2}, l_{3}$ are $K$-linear independent, we get the first case. On the contrary, if these linear forms are $K$-linear dependent, we deduce that $F$ is degenerate.

Let us assume that $F$ is the product of a linear form $l$ and an irreducible quadric $Q$. According to the relative position of $V(l)$ and $V(Q)$, we get the second and the third case.

Let $F$ be a degree three irreducible form. If $C=V(F)$ is singular, then we get the fourth and fifth cases. If $C=V(F)$ is nonsingular, then we may assume that $F=L_{\lambda}$ for $\lambda \in K \backslash\{0,1\}$, i.e., $C$ is an elliptic cubic curve. If $j(\lambda)=0$, then $C$ fits in the orbit of Fermat's curve $y_{1}^{3}+y_{2}^{3}+y_{3}^{3} \equiv 0$, and we get the sixth case. If $j(\lambda) \neq 0$, then it is easy to see that

$$
J=\left(x_{1} x_{2}, H_{1}, H_{2}\right) \subset \operatorname{Ann}_{R}\left(L_{\lambda}\right) .
$$

Since $\lambda^{2}-\lambda+1 \neq 0$, then $J$ is a complete intersection with Hilbert function $\{1,3,3,1\}$. Hence $\left(x_{1} x_{2}, H_{1}, H_{2}\right)=\operatorname{Ann}_{R}\left(L_{\lambda}\right)$.

Remark 3.8. As before, the classification of Artinian Gorenstein $K$-algebras with Hilbert function $\{1,4,4,1\}$ can be obtained by using results on the classification of the degree three hypersurfaces of $\mathbb{P}^{3}$; see, for instance, 3 .

As a consequence of Theorem 3.3, in the particular case of Artinian algebras over a characteristic zero field, we obtain a recent result proved by Henriques and 
Şega on the rationality of the Poincaré series; see [13, Theorem 4.2]. We denote by $P_{K}^{A}(z)$ the Poincare series of $A$, that is

$$
P_{K}^{A}(z)=\sum_{j \geq 0} \operatorname{Tor}_{j}^{A}(K, K) z^{j} .
$$

We recall that a nonzero element $a \in \mathfrak{m}$ is an exact zero-divisor if $(0: a)$ is a principal ideal. Notice that, in the graded case, if $A=A_{F}$ with $F$ a generic cubic form, then an exact zero-divisor always exists and this implies the existence of a Koszul filtration; see [8, Theorem 6.3].

Corollary 3.9 ([13, Theorem 4.2]). Let $A$ be an Artinian Gorenstein local Kalgebra with $\mathfrak{m}^{4}=0$ and $H F_{A}(1)=n \geq 3$. If there exists an exact zero-divisor in $A$, then $A$ is Koszul and hence $P_{K}^{A}(z)$ is rational.

Proof. By the existence of an exact zero-divisor, the Hilbert function of $A$ is balanced, that is, $H F_{A}=\{1, n, n, 1\}$; see [13, Theorem 4.1]. Hence, by Theorem 3.3. $A$ is canonically graded. Because there exists an exact zero-divisor in $G$, by [13, Remark 3.5] and [8, Proposition 2.3 b)] we conclude that $G$ has a Koszul filtration. As a consequence, $A$ is Koszul since $G$ is Koszul, and hence $P_{K}^{A}(z)=P_{K}^{G}(z)$ is rational.

\section{Artinian Gorenstein algebras with socle Degree three}

In [6] Casnati and Notari presented a complete classification of the Artinian Gorenstein local algebras with Hilbert functions $\{1, m, 3,1\}, m \geq 3$. In this section we study the Artinian Gorenstein algebras with Hilbert function $\{1, m, n, 1\}$. By using a result proved by Iarrobino (see [16, Proposition 1.9]), a necessary condition for which the numerical function $\{1, m, n, 1\}$ is the Hilbert function of an Artinian Gorenstein local algebra is that $m \geq n$. If $m=n$, we have proved that every Artinian Gorenstein algebra with Hilbert function $\{1, n, n, 1\}$ is canonically graded. This is no longer true if $m>n$ because the Hilbert function is not symmetric. In this case the associated graded ring $G$ is not Gorenstein, but another Gorenstein graded algebra will play the same role: $Q(0)$ (see Section 2) which is the unique Gorenstein graded quotient of $G$ with the same socle degree. By Iarrobino's work we deduce that

$$
H F_{Q(0)}=\{1, n, n, 1\} .
$$

If we deal with different local rings, we will denote by $Q_{A}(0)$ the module corresponding to the local ring $(A, \mathfrak{m})$.

Theorem 4.1. The following facts are equivalent:

(a) $A$ is an Artinian Gorenstein local K-algebra with Hilbert function $\{1, m, n, 1\}, m>n$

(b) $A \simeq A_{F}$ where $F \in K\left[y_{1}, \ldots, y_{m}\right], F=F_{3}+y_{n+1}^{2}+\cdots+y_{m}^{2}$ with $F_{3}$ a nondegenerate form of degree three in $K\left[y_{1}, \ldots, y_{n}\right]$.

Proof. The part (b) implies (a) is an easy computation based on (3). We prove (a) implies (b). Let $A=R / I$ with $R=K\left[\left[x_{1}, \ldots, x_{n}\right]\right]$, and let $F=F_{0}+F_{1}+F_{2}+F_{3}$ be a polynomial of $P=K\left[y_{1}, \ldots, y_{n}\right]$ of degree three such that $I=\operatorname{Ann}_{R}(F)$ $\left(F_{i}\right.$ denotes the homogeneous components of degree $\left.i\right)$. We know that $Q(0) \simeq$ $R / \operatorname{Ann}_{R}\left(F_{3}\right)$ and it has Hilbert function $\{1, n, n, 1\}$. Since $Q(0)$ is a graded algebra of embedding dimension $n<m$, there exist $L_{n+1}, \ldots, L_{m}$ independent linear 
forms contained in $\operatorname{Ann}_{R}\left(F_{3}\right)$, hence we may assume there exist $L_{1}, \ldots, L_{n}, \ldots, L_{m}$ generators in $P_{1}$ such that $F_{3} \in S=K\left[L_{1}, \ldots, L_{n}\right]$.

Since the Hilbert function of $A$ is $\{1, m, n, 1\}$, and hence $\operatorname{dim}_{K}\left(I^{\perp}\right)_{1}=m$, it is easy to see that

$$
\langle F\rangle_{R}=\left\langle F_{2}+F_{3}\right\rangle_{R}
$$

Now we can write $F_{2}=C+D$ where $C \in K\left[L_{n+1}, \ldots, L_{m}\right]$, and $D$ is a quadratic form in the monomials $L_{i} L_{j}$ with $1 \leq i \leq n, 1 \leq j \leq m$. Since the field $K$ is algebraically closed of characteristic zero, we may assume there exist $\lambda_{i} \in K$ such that $F_{2}=\lambda_{m} L_{m}^{2}+\cdots+\lambda_{n+1} L_{n+1}^{2}+D^{\prime}$ with $D^{\prime}$ the corresponding replacement of $D$.

Since $H F_{A}(1)=m$, we remark that, by (2) and (3) $, \lambda_{m}, \ldots, \lambda_{n+1}$ are different from zero. Summing up this information, we can conclude that there exist $L_{1}, \ldots, L_{n}, \ldots, L_{m}$ independent linear forms of $P$ such that

$$
A \simeq A_{F},
$$

where $F=F_{3}+L_{m}^{2}+\cdots+L_{n+1}^{2}+H$ with $F_{3}$ a homogeneous form of degree three in $K\left[L_{1}, \ldots, L_{n}\right]$ and $H$ a homogeneous form of degree two in the monomials $L_{i} L_{j}$ with $1 \leq i \leq n, 1 \leq j \leq m$.

Since we are considering the linear change of coordinates in $P$ sending $y_{i} \rightarrow L_{i}$, we should replace $R$ and $P$ via the corresponding linear automorphism. For short, we still denote by $R=k\left[\left[x_{1}, \ldots, x_{m}\right]\right]$ and $P=k\left[y_{1}, \ldots, y_{m}\right]$ the corresponding images. Then we have that

$$
A \simeq A_{F},
$$

where $F=F_{3}+y_{n+1}^{2}+\cdots+y_{m}^{2}+H$ with $F_{3}$ a homogeneous form of degree three in $K\left[y_{1}, \ldots, y_{n}\right]$ and $H$ a homogeneous form of degree two in the monomials $y_{i} y_{j}$ with $1 \leq i \leq n, 1 \leq j \leq m$. So we have to prove that, however we fix $H$, there exists an automorphism $\varphi$ of $R / \mathcal{M}^{4}$ which induces

$$
A_{F_{3}+y_{n+1}^{2}+\cdots+y_{m}^{2}} \simeq A_{F_{3}+y_{n+1}^{2}+\cdots+y_{m}^{2}+H}
$$

Let $\varphi$ be the automorphism of $R / \mathcal{M}^{4}$ with the identity as Jacobian defined as

$$
\varphi\left(x_{j}\right)=x_{j}+\sum_{|\underline{i}|=2} a_{\underline{i}}^{j} x^{\underline{i}}
$$

for $j=1, \ldots, m$. We prove that there exists $\underline{a}=\left(a_{\underline{i}}^{1} ; \ldots ; a_{\underline{i}}^{m}\right) \in K^{m\left(\begin{array}{c}n+1 \\ 2\end{array}\right)}$ the vector of the coefficients defining $\varphi$ such that

$$
\left[F_{3}+y_{n+1}^{2}+\cdots+y_{m}^{2}\right]_{E^{*}} M(\varphi)=\left[F_{3}+y_{n+1}^{2}+\cdots+y_{m}^{2}+H\right]_{E^{*}} .
$$

Repeating the same computation as in Theorem 3.3. the matrix associated to $\varphi$, say $M(\varphi)$, is an element of $\mathrm{Gl}_{r}(K), r=\left(\begin{array}{c}n+3 \\ 4\end{array}\right)$, with respect to the basis $E$ of $R / \mathcal{M}^{4}$ ordered by the degree-lexicographic order, hence

$$
M(\varphi)=\left(\begin{array}{l|l|l|l}
1 & 0 & 0 & 0 \\
\hline 0 & I_{m} & 0 & 0 \\
\hline 0 & D & I_{\left(\begin{array}{c}
m+1 \\
2
\end{array}\right)} & 0 \\
\hline 0 & 0 & B & I_{\left(\begin{array}{c}
m+2 \\
3
\end{array}\right)}
\end{array}\right) .
$$

Precisely, $D$ is the $\left(\begin{array}{c}m+1 \\ 2\end{array}\right) \times m$ matrix defined by the coefficients of the degree two monomials of $\varphi\left(x_{j}\right), j=1, \ldots, m$, and $B$ is the $\left(\begin{array}{c}m+2 \\ 3\end{array}\right) \times\left(\begin{array}{c}m+1 \\ 2\end{array}\right)$ matrix defined by the coefficients of the degree three monomials appearing in $\varphi\left(x^{\underline{i}}\right),|\underline{i}|=2$. It 
is clear that $M(\varphi)$ is determined by $D$ and the entries of $B$ are linear forms in the variables $a_{\underline{i}}^{j}$, with $|\underline{i}|=2, j=1, \ldots, m$. Notice that, by the peculiarity of $\varphi$, both $D$ and $B$ have several zero-rows (precisely the rows corresponding to the monomials of degree three divided by $\left.x_{i}, i>n\right)$. Let $H=\sum_{*} \beta_{i j} y_{i} y_{j}$, where $i<j, 1 \leq i \leq n, 1 \leq j \leq m$ and $F_{3}=\sum_{|\underline{i}|=3} \alpha_{\underline{i}} \frac{1}{i \underline{i}} y_{1}^{i_{1}} \cdots y_{n}^{i_{n}}$, hence (10) can be reduced to the equality

$$
\left[\alpha_{\underline{i}}\right] B^{\prime}=\left[\beta_{i j}\right]
$$

where $B^{\prime}$ is the submatrix of $B$ of size $\left(\begin{array}{c}n+2 \\ 3\end{array}\right) \times\left[\left(\begin{array}{c}n+1 \\ 2\end{array}\right)+n(m-n)\right]$ obtained considering the rows and columns corresponding to the degree three monomials in $x_{1}, \ldots, x_{n}$ appearing in $\varphi\left(x_{i} x_{j}\right)$ with $i<j, 1 \leq i \leq n, 1 \leq j \leq m$. Then we get a system of $\left(\begin{array}{c}n+1 \\ 2\end{array}\right)+n(m-n)$ equations which are bihomogeneous polynomials in the $\left\{\alpha_{\underline{i}}\right\}$ and $\underline{a}=\left(a_{i}^{1} ; \ldots ; a_{i}^{m}\right) \in K^{m\left(\begin{array}{c}n+1 \\ 2\end{array}\right)}$ of bidegree $(1,1)$. Then there exists a matrix $M_{F}$ of size $\left[\left(\begin{array}{c}n+1 \\ 2\end{array}\right)+n(m-n)\right] \times m\left(\begin{array}{c}n+1 \\ 2\end{array}\right)$ and entries in the $\left\{\alpha_{\underline{i}}\right\}$ such that

$$
{ }^{t}\left(\left[\alpha_{\underline{i}}\right] B^{\prime}\right)=M_{F}{ }^{t} \underline{a} .
$$

We have to prove that the following linear system in the $\left(\begin{array}{c}n+1 \\ 2\end{array}\right)+n(m-n)$ equations and $m\left(\begin{array}{c}n+1 \\ 2\end{array}\right)$ indeterminates $\underline{a}=\left(a_{\underline{i}}^{1} ; \ldots ; a_{\underline{i}}^{n}\right)$

$$
M_{F}{ }^{t} \underline{a}={ }^{t}\left[\beta_{i}\right]
$$

is compatible. The result follows if we show that $\operatorname{rk}\left(M_{F}\right)$ is maximal, i.e., $\operatorname{rk}\left(M_{F}\right)$ $=\left(\begin{array}{c}n+1 \\ 2\end{array}\right)+n(m-n)$. We will prove that the matrix $M_{F}$ has the following upperdiagonal structure:

$$
\left.M_{F}=\left(\begin{array}{l|l|l|l|l}
M_{F_{3}} & * & \cdots & * & * \\
\hline 0 & \Delta_{F_{3}} & \cdots & * & * \\
\hline \vdots & \vdots & \vdots & \vdots & \vdots \\
\hline 0 & 0 & 0 & \Delta_{F_{3}} & * \\
\hline 0 & 0 & 0 & 0 & \Delta_{F_{3}}
\end{array}\right)\right\} m-n \text { times, }
$$

where

(i) $M_{F_{3}}$ is the $\left(\begin{array}{c}n+1 \\ 2\end{array}\right) \times n\left(\begin{array}{c}n+1 \\ 2\end{array}\right)$ matrix defined in the Claim of Theorem 3.3 ,

(ii) $\Delta_{F_{3}}\left(m-n\right.$ times) is the $n \times\left(\begin{array}{c}n+1 \\ 2\end{array}\right)$ matrix defined in Remark 3.2 of the coefficients of the second derivatives of $F_{3}$.

Following the definition of $B^{\prime}$, we compute $\varphi\left(x_{i} x_{j}\right), i<j, 1 \leq i \leq n, 1 \leq j \leq m$. Hence the entries of the $a_{i}^{l}$-th column of $M_{F}, l=1, \ldots, m$, are the coefficients of the degree three terms in the support of $F_{3}$ which appear in $\varphi\left(x_{i} x_{j}\right)$ with coefficient $a_{\underline{i}}^{l}$.

If $1 \leq i \leq j \leq n$, we are in the same setting of the Claim of Theorem 3.3. and we get $M_{F_{3}}$ corresponding to the $a_{i}^{l}$-th columns with $l=1, \ldots, n$.

We now compute $\varphi\left(x_{i} x_{j}\right)$ with $i=1, \ldots, n$ and $j=n+1, \ldots, m$, then

$$
\varphi\left(x_{i} x_{j}\right)=x_{i} x_{j}+\sum_{|\underline{i}|=2} a_{\underline{i}}^{j} x^{\underline{i}} x_{i}+\sum_{|\underline{i}|=2} a_{\underline{i}}^{l} x^{\underline{i}} x_{j}+\text { terms of degree } 4 .
$$

Since $x^{\underline{i}} x_{j}$ does not appear in the support of $F_{3}$ because $j>n$, then for every $j=n+1, \ldots, m$ we get

$$
\left(M_{F}\right)_{\delta_{i}+\delta_{j}, a_{\underline{i}}^{j}}=\alpha_{\underline{i}+\delta_{i}}
$$


Hence

$$
\left(M_{F}\right)_{\delta_{i}+\delta_{j}, a_{\underline{i}}^{j}}=\left(\Delta_{F_{3}}\right)_{i, \underline{i}}
$$

and (i) and (ii) are proved.

Since $F_{3}$ is nondegenerate for the Hilbert function $\{1, n, n, 1\}$, then by Remark $3.2 \operatorname{rk}\left(\Delta_{F_{3}}\right)=n$ and, from the proof of Theorem $3.3 \operatorname{rk}\left(M_{F_{3}}\right)=\left(\begin{array}{c}n+1 \\ 2\end{array}\right)$. It follows that

as required.

$$
\operatorname{rk}\left(M_{F}\right)=\left(\begin{array}{c}
n+1 \\
2
\end{array}\right)+n(m-n)
$$

We will extend Corollary 3.4 to this more general situation.

Corollary 4.2. There exists an isomorphism between the Artinian Gorenstein local $K$-algebras $(A, \mathfrak{m})$ and $(B, \mathfrak{n})$ with Hilbert function $\{1, m, n, 1\}, m \geq n$, if and only if $Q_{A}(0) \simeq Q_{B}(0)$ as a $K$-algebra.

Proof. If $m=n$, then $Q(0)$ coincides with the associated graded ring and the result follows by Corollary 3.4. Assume $m>n$, then the result follows from Theorem 4.1 which says that the isomorphism classes of $A_{F}$ only depend on $F_{3}$ and hence on the isomorphism classes of $Q(0)$.

The next result extends Corollary 3.5 .

Corollary 4.3. The classification of Artinian Gorenstein $K$-algebras A with Hilbert function $H F_{A}=\{1, m, n, 1\}, m \geq n$, is equivalent to the projective classification of the cubic hypersurfaces $V(F) \subset \mathbb{P}_{K}^{n-1}$, where $F$ is a degree three nondegenerate form in $n$ variables.

By taking advantage of the projective classification of the cubic hypersurfaces in $\mathbb{P}^{r}$, with $r \leq 3$, the above result gives a complete classification of the Artinian Gorenstein local $K$-algebras with Hilbert functions $\{1, m, n, 1\}, n \leq 4$.

\section{ACKNOWLEDGMENTS}

The authors are grateful to A. Conca and G. Valla for useful comments and remarks regarding this work.

\section{REFERENCES}

[1] L. L. Avramov, S. Iyengar, L. M. Şega, Free resolutions over short local rings, J. Lond. Math. Soc. 78 (2008), 459-476. MR2439635 (2009h:13011)

[2] R. Bøgvad, Gorenstein rings with transcendental Poincaré series, Math. Scand. 53 (1983), no. 1, 5-15. MR:733933 (85c:13012)

[3] M. Brundu and A. Logar, Parametrization of the orbits of cubic surfaces, Transform. Groups 3 (1998), 209-239. MR.1640742 (99m:14114)

[4] D. A. Cartwright, D. Erman, M. Velasco, and B. Viray, Hilbert schemes of 8 points, arXiv:0803.0341 (2008).

[5] G. Casnati and R. Notari, On some Gorenstein loci in Hilb $\left(P^{4}\right)$, J. of Algebra 308 (2007). 2055-2074. MR2295072 (2008d:14006)

[6] On the Gorenstein locus of some punctual Hilbert schemes, J. Pure and Appl. Alg. 213 (2009), 2055-2074. MR2533305 (2010g:14003)

[7] G. Casnati, J. Elias, M.E. Rossi, R. Notari, Poincaré series and deformations of Gorenstein local algebras with low socle degree, preprint (2010).

[8] A. Conca, M.E. Rossi, G. Valla, Gröbner flags and Gorenstein Artin rings, Compositio Math. 129 (2001), 95-121. MR 1856025 (2002i:13014) 
[9] J. Elias, G. Valla, Structure theorems for certain Gorenstein ideals. Special volume in honor of Melvin Hochster. Michigan Math. J. 57 (2008), 269-292. MR2492453 (2010i:13020)

[10] _ Isomorphism classes of certain Artinian Gorenstein algebras, Algebras and Representation Theory, DOI 10.1007/s10468-009-9196-8 (2009).

[11] J. Emsalem, Géométrie des points épais, Bull. Soc. Math. France 106 (1978), no. 4, 399-416. MR518046 (80j:14008)

[12] R. Hartshorne, Algebraic geometry, Graduate Texts in Math., 52, Springer-Verlag, 1977. $\operatorname{MR} 0463157(57: 3116)$

[13] I. B. Henriques and L. M. Şega, Free resolutions over short local rings, arXiv: 0904.3510v2 (2009), to appear in Math. Z.

[14] A. Iarrobino, Reducibility of the families of 0-dimensional schemes on a variety. Invent. Math. 15 (1972), 72-77. MR.0301010 (46:170)

[15] _ Compressed algebras: Artin algebras having given socle degrees and maximal length, Trans. Amer. Math. Soc. 285 (1984), no. 1, 337-378. MR748843 (85j:13030)

[16] _ Associated graded algebra of a Gorenstein Artin algebra, Mem. Amer. Math. Soc. 107 (1994), no. 514, viii+115. MR.1184062 (94f:13009)

[17] A. Iarrobino and V. Kanev, Power sums, Gorenstein algebras, and determinantal loci, Lecture Notes in Mathematics, vol. 1721, Springer-Verlag, Berlin, 1999, Appendix C by Iarrobino and Steven L. Kleiman. MR.1735271 (2001d:14056)

[18] I. Kaplanski, Fields and rings, Univ. of Chicago Press (1970).

[19] F.S. Macaulay, The algebraic theory of modular systems, Cambridge University, 1916. MR.1281612(95i:13001)

[20] D.G. Northcott, Injective envelopes and inverse polynomials, J. London Math. Soc. 8 (1972), 290-296. MR0360555 (50:13003)

[21] B. Poonen, Isomorphism types of commutative algebras of finite rank over an algebraically closed field. Computational arithmetic geometry, 111-120, Contemp. Math., 463, Amer. Math. Soc., Providence, RI, 2008. MR 2459993 (2010c:13018)

[22] D.W. Sharpe and P. Vamos, Injective modules, Cambridge Univ. Press (1972). MR0360706 $(50: 13153)$

[23] J. Watanabe, The Dilworth number of Artin Gorenstein rings, Advances in Math. 76 (1989), 194-199. MR1013668 (90j:13023)

Departament d’Àlgebra i Geometria, Universitat de Barcelona, Gran Via 585, 08007 BARCELONA, Spain

E-mail address: elias@ub.edu

Dipartimento di Matematica, Università di Genova, Via Dodecaneso 35, 16146 Genova, ITALY

E-mail address: rossim@dima.unige.it 